\title{
Classical universe emerging from quantum cosmology without horizon and flatness problems
}

\author{
M. Fathi ${ }^{1}$, S. Jalalzadeh ${ }^{1, a}$, P. V. Moniz ${ }^{2,3, b}$ \\ ${ }^{1}$ Department of Physics, Shahid Beheshti University, G. C., Evin, Tehran, Iran \\ ${ }^{2}$ Centro de Matemática e Aplicações-UBI, Covilhã, Portugal \\ ${ }^{3}$ Departmento de Física, Universidade da Beira Interior, 6200 Covilhã, Portugal
}

Received: 16 May 2016 / Accepted: 14 September 2016 / Published online: 27 September 2016

(C) The Author(s) 2016. This article is published with open access at Springerlink.com

\begin{abstract}
We apply the complex de Broglie-Bohm formulation of quantum mechanics in Chou and Wyatt (Phys Rev A 76: 012115, 2007), Gozzi (Phys Lett B 165: 351, 1985), Bhalla et al. (Am J Phys 65: 1187, 1997) to a spatially closed homogeneous and isotropic early universe whose matter contents are radiation and dust perfect fluids. We then show that an expanding classical universe can emerge from an oscillating (with complex scale factor) quantum universe without singularity. Furthermore, the universe obtained in this process has no horizon or flatness problems.
\end{abstract}

\section{Introduction}

In canonical quantum cosmology, the wave function of the universe is obtained from the Wheeler-DeWitt (WDW) equation which is time independent and consequently we have no quantum dynamics. Quantum mechanically speaking, as we know, the Copenhagen interpretation applied to cosmology has some serious problems: The impossibility of a clear division of the total universe into the observer (who measures) and the observed makes it difficult to interpret the wave function of the universe. Moreover, assuming the existence of only one observable universe, the interpretation of the absolute square of the wave function as a probability density is impossible. To find a solution to the above mentioned problems via quantum cosmology, a straightforward and direct way could be the de Broglie-Bohm (dBB), or causal stochastic, interpretation of quantum cosmology. The $\mathrm{dBB}$ interpretation is favorable, especially for a quantum theory of cosmology, because this interpretation is able to resolve the above mentioned conceptual problems of quantum cosmology [4-8]. However, we have a problem in using

\footnotetext{
a e-mail: s-jalalzadeh@sbu.ac.ir

be-mail: pmoniz@ubi.pt
}

the $\mathrm{dBB}$ interpretation in quantum cosmology. It cannot describe the trajectories and non-zero velocities for real wave functions in the minisuperspace (see next section for more details).

In this paper, we propose to look at the problems of standard cosmology from a different and novel quantum cosmological perspective. In recent years, the complex de BroglieBohm (CdBB) formulation of quantum mechanics has been developed as a new alternative interpretation of quantum mechanics [1-3]. It is based on the quantum Hamilton-Jacobi formalism introduced by Leacock and Padgett [9-11]. One of the advantages of this model is that it does not face the problem of stationarity of particles in bound states, encountered in the dBB representation $[12,13]$. The CdBB formulation can be introduced as follows. We employ $\Psi=e^{i S\left(q^{\mu}\right)}$, $S \in \mathbb{C}$, in the corresponding wave equation of the quantum system to obtain a single complex quantum HamiltonJacobi (CQHJ) equation. Since the action $S$ is complexvalued and time remains real-valued, the position and conjugate momentum of particles are complex-valued. In this description [1-3], the transition from a quantum regime to the corresponding classical world occurs for simultaneous very large values of position and quantum numbers of system $[14,15]$, where the quantum force disappears and the particle motion is entirely governed by the classical equation of motion.

In this paper we will investigate, in the CdBB framework, the quantum cosmology of a simple closed FLRW universe, filled with radiation and dust fluids. In Sect. 2, we develop the CQHJ interpretation of our model. We obtain the state dependent quantum cosmological solutions with complex trajectories in complex minisuperspace in Sect. 3. In Sect. 4, we show that for large values of the scale factor and state number $n$, from the model emerges a classical cosmology, without the horizon and flatness problems. 


\section{Complex Bohmian quantum cosmology in minisuperspace}

Simple cosmological models are obtained by considering a class of models in which all but a finite number of degrees of freedom of metric and matter fields are "frozen". This is most commonly achieved by restricting the fields to be homogeneous, so that the line element of spacetime is given by

$\mathrm{d} s^{2}=-N^{2}(t) \mathrm{d} t^{2}+h_{i j}\left(t, x^{j}\right) \mathrm{d} x^{i} \mathrm{~d} x^{j}, \quad i, j=1,2,3$,

where $N(t)$ is the lapse function and the 3 -metric $h_{i j}$ is restricted to be homogeneous. Using the above line element and also assuming the homogeneity of matter fields, the Lagrangian of Einstein-Hilbert plus the matter fields reduces to the minisuperspace form [18]

$$
\begin{gathered}
\mathcal{L}=\frac{1}{2 N} f_{\alpha \beta}\left(q^{\mu}\right) \dot{q}^{\alpha} \dot{q}^{\beta}-N U\left(q^{\mu}\right), \\
\alpha, \beta=0,1,2, \ldots, n-1,
\end{gathered}
$$

where $f_{\alpha \beta}$ is the metric of minisuperspace (a reduced version of the full DeWitt metric) with indefinite signature $(-,+,+,+, \cdots), q^{\alpha}(t)$ denotes local finite coordinates of minisuperspace, and $U\left(q^{\mu}\right)$ is a particularization of $-\sqrt{h} R^{(3)}\left(h_{i j}\right)+V($ Matter $)$, where $V($ Matter $)$ represents the potential terms coming from matter degrees of freedom. Note that sometimes it is convenient to scale the lapse function in terms of the other minisuperspace metric elements (see next section). To obtain the canonical Hamiltonian, we first define the canonical momentum,

$p_{\alpha}=\frac{\partial \mathcal{L}}{\partial \dot{q}^{\alpha}}=f_{\alpha \beta} \frac{\dot{q}^{\beta}}{N}$.

Hence, the canonical Hamiltonian is given by

$H_{c}=p_{\alpha} \dot{q}^{\alpha}-\mathcal{L}=N\left[\frac{1}{2} f^{\alpha \beta} p_{\alpha} p_{\beta}+U\left(q^{\mu}\right)\right]:=N \mathcal{H}$,

where $f^{\alpha \beta}$ is the inverse metric on minisuperspace. The Hamilton equations

$$
\begin{aligned}
& \dot{q}^{\alpha}=\frac{\partial H_{c}}{\partial p_{\alpha}}=N f^{\alpha \beta} p_{\beta}, \\
& \dot{p}_{\alpha}=-\frac{\partial H_{c}}{\partial q^{\alpha}}=-N\left(\frac{1}{2} f_{, \alpha}^{\mu \nu} p_{\mu} p_{\nu}+U_{, \alpha}\right)
\end{aligned}
$$

lead to the field equations

$$
\frac{1}{N} \frac{\mathrm{d}}{\mathrm{d} t}\left(\frac{\dot{q}^{\alpha}}{N}\right)+\frac{1}{N^{2}} \Gamma_{\mu \nu}^{\alpha} \dot{q}^{\mu} \dot{q}^{\nu}+f^{\alpha \beta} U_{, \beta}=0,
$$

where $\Gamma_{\mu \nu}^{\alpha}$ are the components of a Christoffel connection compatible with the metric $f$. In addition, the gauge freedom on choosing a lapse function leads to the following weak equation for a super-Hamiltonian:

$\mathcal{H}=\frac{1}{2} f^{\alpha \beta} p_{\alpha} p_{\beta}+U\left(q^{\mu}\right) \approx 0$.

\subsection{Canonical quantization}

The canonical quantization of this model is accomplished in the coordinate representation, $q^{\alpha}=q^{\alpha}, p_{\alpha}=-i \partial_{\alpha}$ and demanding that the time independent wave function $\Psi\left(q^{\mu}\right)$ is annihilated by the self-adjoint operator corresponding to the Hamiltonian constraint (7), which gives the WDW equation

$\mathcal{H}\left(q^{\alpha},-i \partial_{\alpha}\right) \Psi\left(q^{\mu}\right)=0$.

To solve the operator ordering problem, we should assume that the minisuperspace metric part of WDW equation is covariant under general coordinate transformations in minisuperspace and is also conformally invariant $[19,20]$. Consequently, the WDW equation will be

$\left[-\frac{1}{2} \square+\xi \mathcal{R}+U\left(q^{\mu}\right)\right] \Psi\left(q^{\mu}\right)=0$,

where $\mathcal{R}$ is the Ricci scalar associated to minisuperspace Semi-Riemannian manifold $(f, \nabla), \xi=-\frac{n-2}{8(n-1)}$ for $n \geqslant$ 2 and $\square=f^{\alpha \beta} \nabla_{\alpha} \nabla_{\beta}=\frac{1}{\sqrt{-h}} \partial_{\alpha}\left(\sqrt{-h} f^{\alpha \beta} \partial_{\beta}\right)$ is the D'Alembert operator. Moreover, the covariantly conserved $n$-current corresponding to the WDW equation is given by

$J_{\mu}=\frac{1}{2 i}\left(\Psi^{*} \nabla_{\mu} \Psi-\Psi \nabla_{\mu} \Psi^{*}\right)$.

Note that the WDW equation is a Klein-Gordon type and consequently the probability measure constructed from the above current suffers from the same difficulties with negative probabilities in the usual Klein-Gordon equation.

\section{2 de Broglie-Bohm quantum cosmology}

Before we proceed, some comparisons with dBB approach to quantum cosmology [21-23] will be helpful to explain the necessity of extending the concept of a quantum trajectory to the complex domain.

The WDW equation (9) is separable by means of the general complex assumption (de Broglie ansatz)

$\Psi\left(q^{\mu}\right)=R_{\mathrm{B}}\left(q^{\mu}\right) e^{i S_{\mathrm{B}}\left(q^{\mu}\right)}, \quad R_{\mathrm{B}}$ and $S_{\mathrm{B}} \in \mathbb{R}$.

The subscript " $\mathrm{B}$ " is introduced to highlight that the results are obtained from dBB with CQHJ approach. Substituting (11) into the WDW equation (9) and separating into real and imaginary parts give two coupled non-linear partial differential equations, respectively,

$\frac{1}{2} f^{\alpha \beta} \nabla_{\alpha} S_{\mathrm{B}} \nabla_{\beta} S_{\mathrm{B}}+\xi \mathcal{R}\left(q^{\mu}\right)+U\left(q^{\mu}\right)+Q_{B}\left(q^{\mu}\right)=0$, 
$\nabla_{\alpha} J^{\alpha}=\nabla_{\alpha}\left(f^{\alpha \beta} R_{B}^{2} \nabla_{\beta} S_{\mathrm{B}}\right)=0$,

where

$Q_{B}\left(q^{\mu}\right):=-\frac{\square R_{\mathrm{B}}}{2 R_{\mathrm{B}}}=-\frac{1}{2} \frac{\square|\Psi|}{|\Psi|}$

is the quantum potential. The assumption introduced by the $\mathrm{dBB}$ approach is that we have a well-defined location $q^{\alpha}$ together with $n$-momentum

$p_{\alpha}:=\nabla_{\alpha} S_{\mathrm{B}}=\frac{f_{\alpha \beta}}{N} \dot{q}^{\beta}$.

The lapse function is introduced into the definition of momentum because of the gauge reparameterization freedom of general relativity. It is obvious that for real wave functions $S=0$ and consequently the $n$-momentum (15) vanishes.

\subsection{Complex quantum Hamilton-Jacobi cosmology}

$\mathrm{CQHJ}$ or CdBB mechanics is one of the nine formulations [24] of quantum mechanics, developed along the lines of the classical Hamilton-Jacobi theory. Indeed, it not only provides an alternative interpretation of quantum mechanics but may also serve as a powerful tool to solve quantum mechanical problems [25-28]. The starting point of the CQHJ formalism of quantum mechanics, instead of (11), is to use the following ansatz [9-11]:

$\Psi\left(q^{\alpha}\right)=e^{i S\left(q^{\alpha}\right)}, \quad q^{\alpha}$ and $S\left(q^{\alpha}\right) \in \mathbb{C}$,

where the wave function and the phase are analytically extended to the complex plane by replacing real coordinates $q^{\alpha}$ with complex coordinates, $q^{\alpha}=q_{R}^{\alpha}+i q_{I}^{\alpha}$ though its value will be (physically) meaningful only along the real axis [29,30], and keeping time (and the lapse function) realvalued. Substituting this new ansatz into the WDW equation (9) yields a single equation, known as the $\mathrm{CQHJ}$ equation,

$\frac{1}{2} f^{\alpha \beta} \nabla_{\alpha} S \nabla_{\beta} S+\xi \mathcal{R}\left(q^{\mu}\right)+U\left(q^{\mu}\right)+Q\left(q^{\mu}\right)=0$,

where the new complex quantum potential is given by

$Q:=\frac{1}{2 i} \square S=-\frac{1}{2}\left(\frac{\square \Psi}{\Psi}-\frac{f^{\alpha \beta} \nabla_{\alpha} \Psi \nabla_{\beta} \Psi}{\Psi^{2}}\right)$,

brings all quantum effects into the CQHJ formalism. However, this quantity is not the same as the Bohm quantum potential, defined in (14). Note that there is no expansion in powers of $\hbar$ in the derivation and Eq. (17) is exact. In analogy to standard Bohmian mechanics, complex quantum trajectories can also be defined by analytic continuation of (15) to the complex plane, thus

$p_{\alpha}:=\nabla_{\alpha} S\left(q^{\mu}\right)=\frac{1}{N} f_{\alpha \beta} \dot{q}^{\beta}, \quad p_{\alpha} \in \mathbb{C}$.

Therefore, the main novelty of the CdBB formulation is that now the guidance equation is related to a new complex action function, $S$, and not only to the real part of a wave function. The relationship between the Bohmian momentum (15) and its complex counterpart is

$p_{\alpha}=p_{\alpha}^{(B)}-\frac{i}{R_{\mathrm{B}}} \nabla_{\alpha} R_{\mathrm{B}}$.

This expression explains why it is possible to observe nonvanishing momenta in cases where the Bohmian momenta, $p_{\alpha}^{(B)}$, vanish. In fact, the Bohmian trajectories defined in Eq. (15) only carry information as regards the dynamics of the quantum flow. But the complex quantum trajectories defined in Eq. (20) also include information as regards the probability, because of the following relation between the complex and Bohmian action functions:

$S=S_{\mathrm{B}}-i \ln R_{\mathrm{B}}$.

Therefore, the complex dynamics explains how to get the correct momentum distribution.

Equation (19) is invariant under time reparametrization. To obtain the corresponding field equations, we differentiate the $n$-momentum defined above with respect to cosmic time $t$, which gives $\frac{\mathrm{d} p_{\mu}}{\mathrm{d} t}=\dot{q}^{\alpha} \partial_{\alpha} \partial \mu S=\dot{q}^{\alpha} \partial_{\mu} \nabla_{\alpha} S$. Now, differentiation with respect to cosmic time of Eq. (17) and using the second equality in Eq. (19), we obtain

$\frac{1}{N} \frac{\mathrm{d}}{\mathrm{d} t}\left(\frac{\dot{q}^{\alpha}}{N}\right)+\frac{1}{N^{2}} \Gamma_{\mu \nu}^{\alpha} \dot{q}^{\mu} \dot{q}^{\nu}+f^{\alpha \beta}(U+Q+\xi \mathcal{R})_{, \beta}=0$,

which is the extension of classical field equation (6) to the complex quantum minisuperspace. Furthermore, Eqs. (17) and (19) give us the complex quantum super-Hamiltonian constraint

$\mathcal{H}=\frac{1}{2} f^{\alpha \beta} p_{\alpha} p_{\beta}+\frac{1}{2 i} f^{\alpha \beta} \nabla_{\alpha} p_{\beta}+\xi \mathcal{R}\left(q^{\mu}\right)+U\left(q^{\mu}\right)=0$,

which is a Riccati-like PDE.

The wave function (16) is invariant with respect to a change of its phase $S\left(q^{\mu}\right)$ by an integer multiple of $2 \pi$. Consequently, the definition of momentum (19) gives

$\oint_{C} p_{\mu} \mathrm{d} q^{\mu}=n h=2 \pi n, \quad n=1,2,3, \ldots$,

as a condition of compatibility between the $\mathrm{CQHJ}$ equation (23) and the WDW equation (9). Here, $C$ is a counter clockwise contour in the complex configuration space, enclosing the real line between the classical turning points. Unlike the real-valued equation (12), the CQHJ equation (17) contains all of the information present in the wave function of the universe (16). Some authors have claimed that the $\mathrm{CQHJ}$ formalism is more fundamental than the $\mathrm{dBB}$ interpretation [44]. Moreover, there does not exist an obvious 
probability flux continuity equation in the $\mathrm{CQHJ}$ formalism, as opposed to the coupled equations for the real phase and the real amplitude in the conventional dBB interpretation. However, the most significant difference arises from the fact that for bound states and the real wave functions, the predictions from Bohmian mechanics acquire a new context: For wave functions whose space part is real, the action function in the dBB interpretation, $S_{\mathrm{B}}$, is constant and consequently the velocity field is zero everywhere. In fact, in a Bohmian interpretation of ordinary quantum mechanics, for a stationary bound state, since the $R_{\mathrm{B}}$-amplitude defined in (11) is time independent, the continuity implies that the Bohmian phase, $S_{\mathrm{B}}$, is constant. To solve this problem Floyd $[31,32]$ considered the case that, for this kind of states, the Bohmian phase could be separated into space and time parts, $S(x, t)=W(x)-E t$, where $W(x)$ is the reduced Bohmian action function. Then, Floyd defined the energy dependent modified potential by $U=V+Q_{B}$, where $V$ is the ordinary original potential of wave equation. He pointed out that, for a given eigenvalue of the Schrödinger equation, there is an infinite number of modified potentials $U_{1}, U_{2} \ldots$, and associated with each of these potentials is a trajectory $x_{1}, x_{2} \ldots$ But then the Floydian microstates, $\left\{U_{i}, x_{i}\right\}$, do not arise directly from the Schrödinger equation and this description is not equivalent to the original wave equation, regarding this fact that the microstates provide new dynamical information that is not contained in the Schrödinger equation [29]. But as we know, in quantum gravity and also in quantum cosmology, the general covariance indicates that the wave function is time independent (time problem). Consequently, it is clear that this resolution of the problem is not working in quantum cosmology. This is an undesirable feature in the $\mathrm{dBB}$ interpretation, which claims to make the theory perceivable and causal $[12,13]$. On the other hand, in a CQHJ formulation we can obtain a general velocity field.

Let us further elaborate on the difference of these two approaches with a very simple example from non-relativistic quantum mechanics. Consider the particle in a box model (the infinite square well), which describes a particle free to move in a small one-dimensional space, $0 \leqslant q \leqslant L$, surrounded by impenetrable barriers which is a simple model mainly used to illustrate the differences between classical and quantum mechanics. The space part of the wave function is given by $\psi_{n}(q)=\sqrt{\frac{2}{L}} \sin \left(\frac{n \pi q}{L}\right)$, where $n$ is an integer quantum number. In Bohmian mechanics, comparing this wave function with (11) gives us $S_{\mathrm{B}}=0$ and therefore the momentum of a particle defined by (15) will be zero. On the other hand, in a $\mathrm{CQHJ}$ formulation, according to the definition (16) the action function is given by $S=\ln \left(\sin \left(\frac{n \pi q}{L}\right)\right)$. Therefore, using (19) the momentum of particle will be $p=\frac{\mathrm{d} S}{\mathrm{~d} q}=m \frac{\mathrm{d} q}{\mathrm{~d} t}=-\frac{i n \pi \hbar}{L} \cot \left(\frac{n \pi q}{L}\right)$, where $m$ is the mass of particle. By analytic continuation into the complex space, $q=q_{\mathrm{R}}+i q_{\mathrm{I}}$, where $q_{\mathrm{R}}, q_{\mathrm{I}} \in \mathbb{R}$, and solving the above differential equation, we obtain

$$
\left\{\begin{array}{l}
\cos \left(\frac{n \pi q_{\mathrm{R}}}{L}\right) \cosh \left(\frac{n \pi q_{I}}{L}\right)=C \cos \left(\frac{n^{2} \pi^{2} \hbar t}{m L^{2}}\right), \\
\sin \left(\frac{n \pi q_{\mathrm{R}}}{L}\right) \sinh \left(\frac{n \pi q_{I}}{L}\right)=C \sin \left(\frac{n^{2} \pi^{2} \hbar t}{m L^{2}}\right),
\end{array}\right.
$$

where $C$ is a constant of integration. Hence, the particle has a well-defined complex quantum trajectory with real and imaginary parts satisfying the above equations. Furthermore, the quantum Hamiltonian of a particle will be

$H=\frac{1}{2 m}\left(\frac{\mathrm{d} S}{\mathrm{~d} q}\right)^{2}+Q=\frac{p^{2}}{2 m}+\frac{n^{2} \pi^{2} \hbar^{2}}{2 m L^{2} \sin ^{2}\left(\frac{n \pi q}{L}\right)}=\frac{n^{2} \pi^{2} \hbar^{2}}{2 m L^{2}}$,

where the last equality is obtained by replacing the corresponding complex momentum, $p=-\frac{i n \pi \hbar}{L} \cot \left(\frac{n \pi q}{L}\right)$. Let us now examine the classical limit. For very large values of the quantum number $n$, using the approximate relations $\cosh \left(\frac{n \pi\left|q_{I}\right|}{L}\right)=\sinh \left(\frac{n \pi\left|q_{I}\right|}{L}\right) \simeq \frac{1}{2} \exp \left(\frac{n \pi\left|q_{I}\right|}{L}\right)$ for $n \rightarrow \infty$, the explicit solution of the coupled equations (25) will be

$q_{\mathrm{I}}=\frac{L}{n \pi} \ln (2 C) \simeq 0, \quad q_{\mathrm{R}}=-\frac{n \pi \hbar}{m L} t=p_{c} t$,

where $p_{c}$ is the classical momentum of particle. Therefore, for large values of the quantum number $n$, the imaginary part of the trajectory and momentum will disappear and we will have a classical particle with a real trajectory. The transition from quantum mechanics (CQHJ) to classical mechanics occurs when the motion of the particle falls entirely on the real subspace.

The wave function of the universe is real-valued in many minisuperspace models of the universe [33-38]. To obtain a Bohmian interpretation for these models, the usual procedure is to construct a wave packet by superposition of eigenstates [33-38]. But it is not clear that the hidden symmetries of a model give us permission in general to construct such wave packets [39-42]. On the other hand, the CQHJ interpretation gives us an opportunity to obtain a causal interpretation even for real wave functions of the universe.

\section{FLRW cosmology with a perfect fluid (dust and radiation)}

Let us consider a closed homogeneous and isotropic universe with line element

$\mathrm{d} s^{2}=-N^{2}\left(\eta^{*}\right) \mathrm{d} \eta^{* 2}+a^{2}\left(\eta^{*}\right) \mathrm{d} \Omega^{2}(3)$,

where $N\left(\eta^{*}\right)$ denotes the lapse function, $a\left(\eta^{*}\right)$ is the scale factor, and $d \Omega^{2}{ }_{(3)}$ is the standard line element of a unit 3sphere. The action functional that consists of a gravitational 
part and a matter part when the matter field is considered as a perfect fluid is given by

$$
\begin{aligned}
\mathcal{A}= & \frac{1}{2} M_{\mathrm{P} 1}^{2} \int \sqrt{-g} R \mathrm{~d}^{4} x \\
& +M_{\mathrm{Pl}}^{2} \int_{\partial \mathcal{M}} \sqrt{g^{(3)}} K \mathrm{~d}^{3} x-\int_{\mathcal{M}} \sqrt{-g} \rho \mathrm{d}^{4} x,
\end{aligned}
$$

where $M_{\mathrm{Pl}}^{2}=\frac{1}{8 \pi G}$ is the reduced Planck mass in natural units, $K$ is the trace of the extrinsic curvature of the spacetime boundary, $\rho$ is the total density of matter content of the universe and $\mathcal{M}$ represents the manifold of the spacetime with boundary $\partial \mathcal{M}$. Let us also define some useful quantities. If we assume a universe filled with mixture of noninteracting dust, $\rho_{m}$, and radiation, $\rho_{\gamma}$, then the total energy density will be

$\rho=\rho_{m}+\rho_{\gamma}=\rho_{m i}\left(\frac{a}{a_{i}}\right)^{-3}+\rho_{\gamma i}\left(\frac{a}{a_{i}}\right)^{-4}$,

where $\rho_{m i}$ and $\rho_{\gamma i}$ denote the energy density of dust and radiation, respectively, at initial time $t_{i}$ when the scale factor is $a_{i}=a\left(t_{i}\right)$. Setting the initial time as the GUT time, $t_{i}=$ $t_{\mathrm{GUT}}$, the total energy density (30) can be rewritten as

$\rho=3 H_{g}^{2} M_{\mathrm{Pl}}^{2}\left(\Omega_{m}\left(\frac{a}{a_{g}}\right)^{-3}+\Omega_{\gamma}\left(\frac{a}{a_{g}}\right)^{-4}\right)$,

where $H_{g}$ and $a_{g}$ are the Hubble parameter and scale factor of the universe at the GUT comoving time, $t_{g}$. We also define the density parameter of dust and radiation at the GUT epoch by $\Omega_{m}=\Omega_{m}\left(t_{g}\right)=\rho_{m}\left(t_{g}\right) /\left(3 H_{g}^{2} M_{\mathrm{Pl}}^{2}\right)$ and $\Omega_{\gamma}=\Omega_{\gamma}\left(t_{g}\right)=$ $\rho_{m}\left(t_{g}\right) /\left(3 H_{g}^{2} M_{\mathrm{Pl}}^{2}\right)$. In addition, if we redefine the scale factor, the lapse function and time coordinate,

$\left\{\begin{array}{l}q=\frac{a}{a_{g}}-\frac{\Omega_{m}}{2\left|\Omega_{k}\right|}, \\ N=\frac{a}{a_{g}} \tilde{N}, \\ \mathrm{~d} \eta=H_{g} \mathrm{~d} \eta^{*},\end{array}\right.$

and introduce the following parameters:

$\left\{\begin{array}{l}M=\frac{12 \pi^{2} M_{\mathrm{Pl}}^{2}}{H_{g}^{2}\left|\Omega_{k}\right|^{\frac{3}{2}}}, \quad \omega=\sqrt{\left|\Omega_{k}\right|}, \\ \mathcal{E}=\frac{M}{2}\left[\frac{\Omega_{m}^{2}}{4\left|\Omega_{k}\right|}+\Omega_{\gamma}\right]\end{array}\right.$

where $\Omega_{k}=-\frac{1}{a_{g}^{2} H_{g}^{2}}$ denotes spatial curvature density, at the GUT epoch, then the total Lagrangian of the model in one-dimensional minisuperspace will be

$\mathcal{L}=\frac{1}{2} \frac{M}{\tilde{N}} \dot{q}^{2}-\frac{1}{2} M \tilde{N} \omega^{2} q^{2}+\tilde{N} \mathcal{E}$,

where a dot denotes the derivative with respect to $\eta$. The conjugate momentums of $q$ and the lapse function $\tilde{N}$ are

$p=\frac{M}{\tilde{N}} \dot{q}, \quad p_{\tilde{N}}=0$.
The canonical Hamiltonian (4) for this model will be

$H_{c}=\dot{q} p+p_{\tilde{N}} \dot{\tilde{N}}-\mathcal{L}=\tilde{N}\left(\frac{p^{2}}{2 M}+\frac{1}{2} M \omega^{2} q^{2}-\mathcal{E}\right)$.

Because of the existence of constraint $p_{\tilde{N}}=0$, the Lagrangian is singular. Hence, the total Hamiltonian could be constructed by adding to the Hamiltonian (36) the primary constraint, multiplied by an arbitrary function of time, $\lambda(\eta)$

$H_{T}=N\left(\frac{p^{2}}{2 M}+\frac{1}{2} M \omega^{2} q^{2}-\mathcal{E}\right)+\lambda p_{\tilde{N}}$.

The requirement that the primary constraint, $p_{\tilde{N}}=0$, must hold during the evolution means that

$\dot{p}_{\tilde{N}}=\left\{p_{\tilde{N}}, H_{T}\right\} \approx 0$.

Equations (37) and (38) lead to the secondary constraint

$\mathcal{H}=\frac{p^{2}}{2 M}+\frac{1}{2} M \omega^{2} q^{2}-\mathcal{E} \approx 0$,

which is a weak equation for the super-Hamiltonian defined in Eq. (7).

\subsection{Brief discussion of the classical minisuperspace}

The Hamilton equations of motion (5), in the gauge $\tilde{N}=1$, will be

$\dot{q}=\frac{p}{M}, \quad \dot{p}=-M \omega^{2} q$

which lead us to solution $q=A \cos (\omega \eta+\theta)$, where $A$ and $\theta$ are constants of integration. The super-Hamiltonian constraint (39) fixes the value of $A$ as $A=\frac{1}{\omega} \sqrt{\frac{2 \mathcal{E}}{M}}$. If we assume that the initial singularity occurs at $\eta=0$, and by using the relation $a_{g} H_{g} \mathrm{~d} t=a \mathrm{~d} \eta$ defined in (32) between cosmic time $t$ and conformal time $\eta$, the scale factor in terms of the comoving cosmic time $t$ will be

$\left\{\begin{array}{l}a(t)=\frac{a_{\mathrm{Max}}}{1-\sec (\theta)}[\sec (\theta) \cos (\omega \eta+\theta)-1], \\ t=\frac{\Omega_{m}}{2 H_{g}\left|\Omega_{k}\right|}\left[\eta-\frac{1}{\omega} \sec (\theta) \sin (\omega \eta+\theta)\right],\end{array}\right.$

where $\cos (\theta):=-\frac{\Omega_{m}}{2 \sqrt{\left|\Omega_{k}\right|}} \sqrt{\frac{M}{2 \mathcal{E}}}$ and $a_{\mathrm{Max}}:=\frac{a_{g}}{\sqrt{\left|\Omega_{k}\right|}} \sqrt{\frac{2 \mathcal{E}}{M}}+$ $\frac{a_{g} \Omega_{m}}{2\left|\Omega_{k}\right|}$ is the maximum value of scale factor. It is easy to see that, at the GUT epoch, the super-Hamiltonian constraint (Friedmann equation) reduces to the well-known relation between the energy density parameters,

$\Omega_{\gamma}+\Omega_{m}+\Omega_{k}=1$.

\subsection{FLRW quantum cosmology with a perfect fluid}

The standard canonical quantization of this simple model is accomplished straightforwardly in the coordinate representation $q=q$ and $p=-i \frac{\mathrm{d}}{\mathrm{d} q}$. Then the Hamiltonian constraint 
(39) becomes the WDW equation for the wave function of the universe,

$$
\left(-\frac{1}{2 M} \frac{\mathrm{d}^{2}}{\mathrm{~d} q^{2}}+\frac{1}{2} M \omega^{2} q^{2}\right) \Psi_{n}(q)=\mathcal{E}_{n} \Psi_{n}(q) .
$$

The eigenvalues and normalized eigenfunctions are

$$
\left\{\begin{array}{l}
\mathcal{E}_{n}=\left(n+\frac{1}{2}\right) \omega \\
\psi_{n}(q)=\frac{1}{\sqrt{2^{n} n !}}\left(\frac{M \omega}{2}\right)^{\frac{1}{4}} e^{-\frac{M \omega}{2} q^{2}} H_{n}(\sqrt{M \omega} q),
\end{array}\right.
$$

where $H_{n}(x)$ denotes the Hermite polynomials. Substituting $\mathcal{E}$ and $\omega$ defined by (33) into the eigenvalue equation obtained in Eq. (44), we obtain the following relation between the energy density parameters:

$\frac{1}{\left|\Omega_{k}\right|^{2}}\left(\frac{\Omega_{m}^{2}}{4\left|\Omega_{k}\right|}+\Omega_{\gamma}\right)=\frac{H_{g}^{2}}{6 \pi^{2} M_{\mathrm{Pl}}^{2}}\left(n+\frac{1}{2}\right)$,

or equivalently

$\left|\Omega_{k}\right|^{3}-\frac{6 \pi^{2} M_{\mathrm{Pl}}^{2}}{\left(n+\frac{1}{2}\right) H_{g}^{2}} \Omega_{\gamma}\left|\Omega_{k}\right|-\frac{3 \pi^{2} M_{\mathrm{Pl}}^{2}}{2\left(n+\frac{1}{2}\right) H_{g}^{2}} \Omega_{m}^{2}=0$,

which is the quantum cosmological counterpart of the classical relation (42). In ordinary quantum mechanics, the transition to excited states may occur induced through a "time dependent" term present in the Hamiltonian. But in general relativity and subsequent quantum cosmology, we have general covariance and general invariance of field equations. In our simple case, there is not any explicitly time dependent Lagrangian or Hamiltonian. Moreover, to have a change in the value of the quantum number, $n$, we would need some dynamics in the quantum cosmology to make such a change. For example, in quantum mechanics, if we consider the superposition of states, then there is a possibility of time changing between various states on the superposition. But in strict quantum cosmology, we do not have any explicit time. Only through e.g., some quantum to classical transition and a decoherence process [43], a WKB time may emerge, through the presence of fluctuations in the matter field, for example. In our model, there is an Hamiltonian constraint. In addition, because of the non-linearity of the field equations (17), the superposition of the wave functions will not be a solution of (17).

Let us further add that, in our model, the quantum number $n$ is related to the matter content of the universe, or equivalently, to the entropy of radiation [16]. Hence, changing the value of $n$ would be equivalent to a change in the matter content of the universe. But in our model that is not consistent with covariant conservation of the fluid. But instead, if quantum matter fields would be present in a similar CdBB model we can investigate this aspect in an suitable context, which we leave for subsequent work.

\section{$4 \mathrm{CQHJ}$ formulation for the FLRW with a perfect fluid}

As we saw in Eq. (16), the starting point of the CQHJ formulation is the insertion of the ansatz

$\Psi(q)=e^{i S(q)}$,

in the WDW equation (43), where the wave function and the phase are analytically extended to the complex plane by replacing the real coordinate of minisuperspace $q$ with a complex coordinate and keeping time (and the lapse function) real-valued. By inserting Eq. (47) into the WDW equation (43) we obtain

$\frac{1}{2 M}\left(\frac{\mathrm{d} S}{\mathrm{~d} q}\right)^{2}+\frac{1}{2} M \omega^{2} q^{2}-i \frac{1}{2 M} \frac{\mathrm{d}^{2} S}{\mathrm{~d} q^{2}}-\mathcal{E}=0$.

The guidance equation for complex quantum trajectories (19) gives the momentum

$p=\frac{M}{\tilde{N}} \frac{\mathrm{d} q}{\mathrm{~d} \eta}:=\frac{\mathrm{d} S}{\mathrm{~d} q}=-i \frac{\mathrm{d}}{\mathrm{d} q} \ln (\Psi)$,

where $(q, p) \in \mathbb{C} \times \mathbb{C}$. As we saw in the previous section, this means that the coordinate of minisuperspace, $q$, has been replaced by a complex variable $q=q_{\mathrm{R}}+i q_{\mathrm{I}}$, where $q_{\mathrm{R}}, q_{\mathrm{I}} \in$ $\mathbb{R}$. Using Eq. (49) in Eq. (48), we obtain the complex quantum super-Hamiltonian constraint

$\mathcal{H}_{Q}=\frac{p^{2}}{2 M}+\frac{1}{2} M \omega^{2} q^{2}+Q(\Psi)-\mathcal{E}=0$,

where

$Q(\Psi)=\frac{1}{2 M i} \frac{\mathrm{d} p}{\mathrm{~d} q}=-\frac{1}{2 M} \frac{\mathrm{d}^{2}}{\mathrm{~d} q^{2}} \ln (\Psi(q))$

denotes the complex quantum potential. Equation (50) is a Riccati differential equation for the complex quantum momentum. The Hamilton equations of motion for the quantum state $\Psi_{n}$ can be derived from the quantum superHamiltonian (50) (in gauge $\tilde{N}=1$ ):

$\left\{\begin{array}{l}\dot{q}=\frac{\partial \mathcal{H}}{\partial p}=\frac{p}{M}, \\ \dot{p}=-\frac{\partial \mathcal{H}}{\partial q}=-M \omega^{2} q-\frac{d Q}{d q},\end{array}\right.$

where a dot denotes a derivative with respect to $\eta$. Consequently, the complex quantum Friedmann and the Raychaudhuri equations will be

$\left\{\begin{array}{l}\frac{1}{2} M \dot{q}^{2}+\frac{1}{2} M \omega^{2} q^{2}+Q\left(\Psi_{n}\right)-\mathcal{E}=0, \\ M \ddot{q}=-M \omega^{2} q-\frac{\mathrm{d} Q\left(\Psi_{n}\right)}{\mathrm{d} q} .\end{array}\right.$

\subsection{Trajectories in the CQHJ formulation}

Let us elaborate on how the CQHJ can be applied to extract solutions.

Before dealing with an observable universe, let us study in detail two ground state and first excited universes. We start 
as a example with the ground state universe, $n=0$, with eigenvalue $\mathcal{E}_{0}=\frac{\omega}{2}$, obtained from Eq. (44). In this case, the quantum potential (51) will be $Q=\frac{\omega}{2}$. Moreover, from Eqs. (49), (52), and $\Psi_{0}=C_{0} \exp \left(-\frac{M \omega}{2} q^{2}\right)$, we obtain

$p=M \dot{q}=i M \omega q$,

with the solution $q=q_{\mathrm{R}}+i q_{\mathrm{I}}=A \exp [i(\omega \eta+\theta)]$ where $A, \theta \in \mathbb{R}$. Note that the value of $A$, unlike the classical case, cannot be fixed by the quantum super-Hamiltonian (50). If we insert the quantum potential and complex conjugate variables $(q, p)$ into the constraint equation, it gives us only the eigenvalue of the ground state. According to Eq. (32), the real part of $q$ is related to the scale factor via $q_{\mathrm{R}}=$ $\frac{a}{a_{g}}-\frac{\Omega_{m}}{2\left|\Omega_{k}\right|}$. Furthermore, the conformal time $\eta$ and cosmic time $t$ are related by $a_{g} H_{g} \mathrm{~d} t=a(\eta) \mathrm{d} \eta$ as in the classical case, because complex quantum variables are confined to the minisuperspace, according to the quantization rule, and the lapse function and all time coordinates are real. Therefore, the scale factor will be

$$
\left\{\begin{array}{l}
a(t)=a_{g}\left(A \cos (\omega \eta+\theta)+\frac{\Omega_{m}}{2\left|\Omega_{k}\right|}\right), \\
t=\frac{1}{H_{g}}\left(\frac{A}{\omega} \sin (\omega \eta+\theta)+\frac{\Omega_{m}}{2\left|\Omega_{k}\right|} \eta\right) .
\end{array}\right.
$$

From (55), using the initial conditions $a\left(t_{g}\right)=a_{g}$ and $H_{g}=$ $H\left(t_{g}\right)=\left.\frac{1}{a} \frac{d a}{d t}\right|_{t=t_{g}}$, we obtain

$$
A=\frac{1}{\sqrt{\left|\Omega_{k}\right|}} \sqrt{1-\Omega_{k}-\Omega_{m}-\Omega_{\gamma}+\frac{H_{g}^{2} \Omega_{k}^{2}}{12 \pi^{2} M_{\mathrm{Pl}}^{2}}} .
$$

Note that according to Eq. (46) $1-\Omega_{k}-\Omega_{m}-\Omega_{\gamma} \neq 0$ in quantum cosmology. We also find the following relation between the scale factor and the imaginary part:

$$
\left(\frac{a}{a_{g}}-\frac{\Omega_{m}}{2\left|\Omega_{k}\right|}\right)^{2}+q_{\mathrm{I}}^{2}=A^{2} \text {. }
$$

A point to be noticed is that the real part of the scale factor obtained in Eq. (55) is similar to the classical motion of the closed universe (40), but in the quantum derived expression for the universe, (55), the maximum of scale factor is given by $a_{\mathrm{Max}}:=a_{g} A+\frac{a_{g} \Omega_{m}}{2\left|\Omega_{k}\right|}$ and the imaginary part of the motion is not negligible at all and a universe with $n=0$ is entirely in the quantum domain. Another interesting feature is that

$\frac{1}{2} M \omega^{2} q^{2}+\frac{p^{2}}{2 M}=0, \quad Q=\frac{\omega}{2}$,

which indicates that the dynamics of such a universe completely originates from the quantum potential. The solution $q=q_{\mathrm{R}}+i q_{\mathrm{I}}=A \exp [i(\omega \eta+\theta)]$ shows that the zero-mode universe is not singular. Moreover, we can easily show that the real part of the solution (55) is not singular for $\Omega_{m}>1$. We will show that for universes with very large values of the quantum number $n$, the quantum potential vanishes and the model reduce to a classical universe.
Before dealing with this classical limit of our model, let us consider a universe with $n=1$ as a second example.

To obtain the trajectory for the $n=1$ quantum universe, we apply $\Psi_{1}=C_{1} y \exp \left(-\frac{M \omega}{2} q^{2}\right)$ to the definition of momentum in Eq. (49), which leads to

$p=M \dot{q}=i\left(M \omega q-\frac{1}{q}\right)$.

The integration gives the eigen-trajectory

$M \omega q^{2}=1+A e^{i(2 \omega \eta+\theta)}$,

where $A, \theta \in \mathbb{R}$. The real part of (60) together with the relation $q_{\mathrm{R}}=\frac{a}{a_{g}}-\frac{\Omega_{m}}{2\left|\Omega_{k}\right|}$ gives

$$
\begin{aligned}
& \frac{a}{a_{g}}=\frac{\Omega_{m}}{2\left|\Omega_{k}\right|} \\
& +\frac{1}{\sqrt{2 M \omega}}\left(1+A \cos (2 \omega \eta+\theta)+\sqrt{A^{2}+1+2 A \cos (2 \omega \eta+\theta)}\right)^{\frac{1}{2}} .
\end{aligned}
$$

The value of $A$ can be calculated from the initial values $a\left(t_{g}\right)=a_{g}$ and $H\left(t_{g}\right)=H_{g}$, similar to the case of the ground state.

A more complete understanding of the dynamics in complex minisuperspace is gained from the consideration of the complex Raychaudhuri equation. Inserting $\Psi_{1}$ into the Raychaudhuri equation in (53) gives

$M \ddot{q}=-M \omega^{2} q+\frac{1}{M} q^{-3}$.

When $|q| \gg \frac{1}{\sqrt{M \omega}}$, the quantum force $-\frac{\mathrm{d} Q}{\mathrm{~d} q}=\frac{1}{M q^{3}}$ approaches zero and the classical equation of motion is recovered. When $|q| \ll \frac{1}{\sqrt{M \omega}}$, the classical force becomes negligible and the motion is dominated by the quantum force. Figure 1 shows the complex paths in minisuperspace for the $n=1$ universe. This universe is non-singular like the $n=0$ universe.

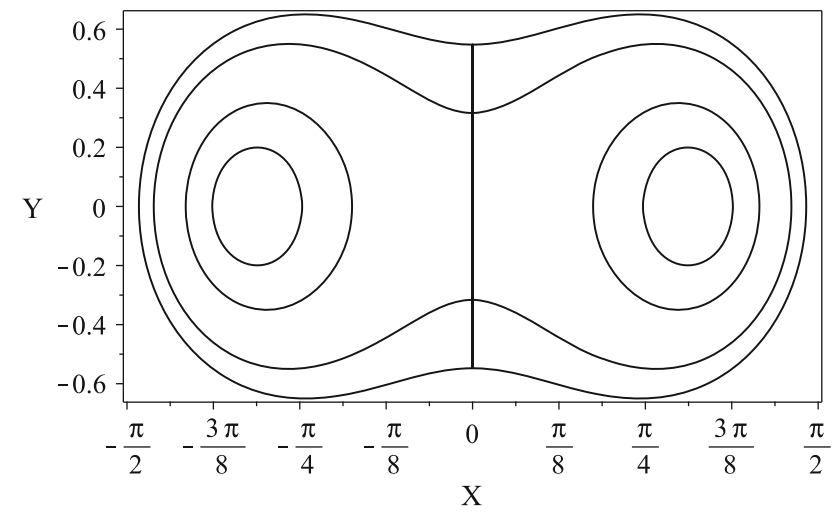

Fig. 1 Complex paths in complex minisuperspace for $n=1$ where we defined $X:=\sqrt{M \omega} q_{\mathrm{R}}$ and $Y:=\sqrt{M \omega} q_{\mathrm{I}}$. The contours are plotted for $\omega=0.5, A=0.4,0.7,1.1$, and 1.3 values 


\subsection{Emergence of a classical universe}

Let us now investigate the behavior of the model for very large values of the quantum state number $n$. Let us first estimate the value of the quantum number $n$ for our universe.

In Ref. [16], it was showed that the total entropy for radiation in the model investigated herein is given by

$S_{\gamma}=1.3 g^{\frac{1}{4}}\left(n+\frac{1}{2}\right)^{\frac{3}{4}}$,

where $g$ is the internal degrees of freedom of radiation. This shows that the value of the quantum number $n$ is related to the matter content of the universe. For a universe created from nothing with a large amount of matter, the quantum number is also large; and inversely, for a universe with large quantum number $n$, the matter content of that universe is also great. On the other hand, the entropy of radiation in the observable part of the universe is about $10^{88}$ [17]. Consequently, this allows us to estimate the value of the quantum number in our universe as $n \gtrsim 10^{118}$.

For a large quantum number $n$, the wave function (44) has the following asymptotic expansion:

$\Psi_{2 n+1}(y)=C_{2 n+1} \sin (\sqrt{2 M n \omega} y)$.

Therefore, the complex quantum potential (51) will be

$Q=\frac{2 n \omega}{\sin ^{2}(\sqrt{2 M n \omega q})}$.

Moreover, using (49) and (52), the equation of motion will be

$M \dot{q}= \begin{cases}i \sqrt{2 M n \omega} \cot (\sqrt{2 M n \omega} q), \sqrt{2 M n \omega}|q| \gg 1, \\ i\left(2 M n \omega q-\frac{1}{q}\right), & \sqrt{2 M n \omega}|q| \ll 1 .\end{cases}$

The integration for large values of $q$ gives the eigentrajectory, thus

$\cos (\sqrt{M n \omega} q)=A e^{-i(2 n \omega \eta+\theta)}$,

where $A, \theta \in \mathbb{R}$. Separating the real and imaginary parts of $q=q_{\mathrm{R}}+i q_{\mathrm{I}}$ gives us

$\left\{\begin{array}{l}e^{\beta} \cos \alpha=2 A \cos (2 n \omega \eta+\theta), \\ e^{\beta} \sin \alpha=2 A \sin (2 n \omega \eta+\theta),\end{array}\right.$

where $\alpha=\sqrt{2 M n \omega} q_{\mathrm{R}}$ and $\beta=\sqrt{2 M n \omega} q_{\mathrm{I}}$.

The solution of the above equations, using the definition of $M$ and $\omega$ in (33) and the relation between conformal time and comoving time, $a_{g} H_{g} \mathrm{~d} t=a \mathrm{~d} \eta$, yields

$\frac{a(t)}{a_{g}}=\left(\frac{2 n}{3}\right)^{\frac{1}{4}}\left(\frac{H_{g}^{2}\left|\Omega_{k}\right|}{\pi M_{\mathrm{Pl}}}\right)^{\frac{1}{2}} \sqrt{t}$ and

$q_{\mathrm{I}}=\frac{\ln (2 A)}{\sqrt{4 n M \omega}}$.

Equation (70) implies that for very large values of quantum number, $n \gg 1$, the imaginary part of the motion vanishes and the trajectory falls entirely on the real axis of minisuperspace. Inserting the initial condition $H\left(t_{g}\right)=H_{g}$ in the time derivative of Eq. (69) gives $2 H_{g} t_{g}=1$. Furthermore, the initial condition $a\left(t_{g}\right)=a_{g}$ gives

$\left|\Omega_{k}\right|=\frac{\pi M_{\mathrm{Pl}}}{H_{g}}\left(\frac{6}{n}\right)^{\frac{1}{2}}=2 \pi \frac{t_{g}}{t_{\mathrm{Pl}}}\left(\frac{6}{n}\right)^{\frac{1}{2}}$,

where $t_{\mathrm{Pl}}=1 / M_{\mathrm{Pl}}$ denotes Planck's time in natural units. If we insert Eq. (71) into (46) we will obtain the energy density parameter,

$\Omega_{m}=\left(\frac{8 \pi t_{g}}{t_{\mathrm{Pl}}}\left(1-\Omega_{\gamma}\right)\right)^{\frac{1}{2}}\left(\frac{6}{n}\right)^{\frac{1}{4}}$

On the other hand, for $\sqrt{2 M n \omega}|q| \ll 1$ the motion is dominated by the quantum force at the very early universe, where according to the first equation in Eq. (66), the eigen-trajectory for very small values of $q$, similar to Eq. (61) is oscillatory and non-singular in an initial moment, $t=0$, with minimum

$a(0)=\frac{\Omega_{m}}{2\left|\Omega_{k}\right|} a_{g}$

Inserting the quantities $\left|\Omega_{k}\right|$ and $\Omega_{m}$ obtained above in Eqs. (71) and (72) gives

$\Omega_{\gamma}=1-\frac{2 \pi t_{g}}{t_{\mathrm{Pl}}}\left(\frac{a(0)}{a_{g}}\right)^{2}\left(\frac{6}{n}\right)^{\frac{1}{2}}$.

Inserting again the energy density parameter of radiation obtained in the above equation into Eq. (72) gives

$\Omega_{m}=\frac{4 \pi t_{g}}{t_{\mathrm{Pl}}} \frac{a(0)}{a_{g}}\left(\frac{6}{n}\right)^{\frac{1}{2}}$.

\subsection{Classical implications from CQHJ}

The grand unification epoch could have ended at approximately $t_{g} \simeq 10^{-36} \mathrm{~s}$ after the Big Bang. Moreover, the quantum description of the universe is that of a non-singular scenario with initial scale factor $a(0)$ as indicated in (73).

\subsubsection{On the flatness issue}

If we assume the initial value of scale factor at the beginning of Planck's length, $a(0) \simeq 10^{-33} \mathrm{~cm}$, and take $n \gtrsim 10^{118}$ as estimated in previous section, then, according to Eq. (71) 
the spatial curvature parameter at the GUT phase transition time will be

$\left|\Omega_{k}\right| \lesssim 10^{-58}$.

Using the definition of the curvature parameter $\left|\Omega_{k}\right|=\frac{1}{a_{g}^{2} H_{g}^{2}}$, the relation $2 H_{g} t_{g}=1$, and Eq. (76), we obtain the linear size of the universe at GUT time,

$a_{g} \simeq 1 \mathrm{~mm}$.

Now, inserting these values into Eqs. (74) and (75) gives us

$\Omega_{m} \lesssim 10^{-78}, \quad \Omega_{\gamma} \simeq 1-10^{-86}$.

In other words, according to Eq. (66), for very large values of $n$ and $\sqrt{2 M \omega n}|q| \ll 1$, or equivalently, for scale factors smaller than $a_{g}$, the model predicts an oscillating quantum universe, where the minisuperspace is complex and without initial Big Bang singularity, while for $a \gtrsim a_{g}$ the emerging universe is completely classical with real minisuperspace, is very close to spatially flatness, and is radiation dominated with a scale factor given by

$a(t)=a_{g} \sqrt{\frac{t}{t_{g}}}$,

where the density of matter is lower by many orders of magnitude.

\subsubsection{On the horizon issue}

According to the CMB observations the whole of the universe was causally connected at last scattering surface time [45]. But in standard FLRW classical cosmology, the universe is causally connected by an angle of order unity, which is in conflict with observation.

The necessary condition for the universe to be causally connected at time $t$ is

$\mathrm{d}_{H}(t)=a(t) \int_{0}^{t} \frac{\mathrm{d} t^{\prime}}{a\left(t^{\prime}\right)} \geq \mathrm{d}_{p}(t)=a(t) \int_{0}^{r_{\mathrm{Max}}} \frac{\mathrm{d} r}{\sqrt{1-k r^{2}}}$,

where $\mathrm{d}_{H}$ and $\mathrm{d}_{p}$ represent the horizon and proper distances at time $t$, respectively.

For open and flat universes the right hand side of Eq. (80) becomes infinite for $r_{\text {Max }}=\infty$ and therefore the global causality failed at any finite time. On the other hand, for a closed universe, $r_{\text {Max }}$ is finite and we can define a causal time $\left(t_{\text {cau }}\right)$ at which the whole of the universe becomes causally connected,

$\int_{0}^{t_{\mathrm{cau}}} \frac{\mathrm{d} t^{\prime}}{a\left(t^{\prime}\right)}=\int_{0}^{1} \frac{\mathrm{d} r}{\sqrt{1-r^{2}}}=\frac{\pi}{2}$.

To calculate the left-hand side of (81) we assume that the classical universe started just after the moment $t_{g}$, and con- sequently we take the lower bound of integration as the GUT time. Hence, we will obtain

$t_{\text {cau }}=\left(\frac{\pi}{4}+1\right)^{2} t_{g}$,

which shows that the whole of the universe becomes causally connected at the GUT phase transition time, because of quantum effects of gravity at very early universe, in the rather specific and simple model we have been exploring.

\section{Conclusions and discussion}

In this paper, we have introduced a simple quantum cosmological model to which we applied the quantum HamiltonJacobi formalism with the concept of a complex quantum trajectory [1-3].

Our purpose was to address from a new and different perspective some problems of the standard Big Bang setting of cosmology. This scenario, based on a matter content described by dust and radiation, is observationally successful in describing the present epoch of the universe and up to some time into the past. From the microwave background radiation it is possible to trace it up to a red-shift $z \sim 10^{3}$, while nucleosynthesis probes it up to $z \sim 10^{11}$. We do not have observational evidence regarding the correctness of this scenario at larger red-shifts, for example the standard GUT area, $z \sim 10^{27}$.

On the other hand, theoretical inconsistencies of scenario, like the existence of an initial singularity and also the flatness and horizon problems, definitely suggest the breakdown of this framework at some large red-shifts. Cosmological inflation is a mechanism that improves on the problems mentioned.

In this paper, we have nevertheless explored the ability of quantum cosmology to provide a new insight on those problems, without inserting explicitly any new set of fields, parameters or extensions. Our new tool is the CdBB framework.

We considered a closed FLRW universe filled with a dust fluid and radiation. We showed that for very large values of a quantum state number $n$, which according to Eq. (63) is related to the entropy of radiation in the universe, the classical universe can emerge from an oscillating complex quantum universe, without singularity, horizon, and flatness problems.

As a final note, let us add that it would be of significant interest to extend this work to other models; namely, either a anisotropic model ${ }^{1}$ or a model with a scalar field. Furthermore, one could address a setting in which inhomogeneities are allowed to be present perturbatively. Dealing with inho-

\footnotetext{
1 Viz. Bianchi type-I or even type-IX, to discuss eventual emergent chaotic behavior [46].
} 
mogeneous perturbations will be of relevance because different interpretations of quantum mechanics may have different observational consequences. Specifically, this is so on choosing to employ (in the central role) the "collapse of the wave function" toward the prediction of the spectrum of perturbations (cf. in particular [47]). As far as the usual dBB approach to quantum cosmology is concerned, linear cosmological perturbations have been considered (see the references in [48-51]). Falsifiable observational consequences were pointed out and some fitted with known data, although others remain to be tested. In the dBB theory of quantum cosmology, desirable fluctuations (inhomogeneities in the matter fields densities) do occur, and the undesirable fluctuations (Boltzmann brains in the late universe) presumably do not occur, because there are no external observers causing the wave function to collapse [52]. Regarding CdBB into quantum cosmology, as introduced and explored in this paper, that remains an open issue to contemplate. We are leaving the above enticing research lines for future work.

Acknowledgments The authors would like to sincerely thank the anonymous referees for constructive and helpful comments to improve the original manuscript. P.V. Moniz research work is supported by the Portuguese grant UID/MAT/00212/2013.

Open Access This article is distributed under the terms of the Creative Commons Attribution 4.0 International License (http://creativecomm ons.org/licenses/by/4.0/), which permits unrestricted use, distribution, and reproduction in any medium, provided you give appropriate credit to the original author(s) and the source, provide a link to the Creative Commons license, and indicate if changes were made. Funded by SCOAP ${ }^{3}$.

\section{References}

1. C.C. Chou, R.E. Wyatt, Phys. Rev. A 76, 012115 (2007)

2. E. Gozzi, Phys. Lett. B 165, 351 (1985)

3. R.S. Bhalla, A.K. Kapoor, P.K. Panigrahi, Am. J. Phys. 65, 1187 (1997)

4. F.G. Alvarenga, J.C. Fabris, N.A. Lemos, G.A. Monerat, Gen. Rel. Grav. 34, 651 (2002). arXiv:gr-qc/0106051

5. F. Shojai, A. Shirinifard, Int. J. Mod. Phys. D 14, 1333 (2005). arXiv:gr-qc/0504138

6. N. Pinto-Neto, J.C. Fabris, Class. Quantum Grav. 30, 143001 (2013). arXiv: 1306.0820

7. P. Pedram, S. Jalalzadeh, Phys. Lett. B 660, 1 (2008). arXiv:0712.2593

8. P. Peter, N. Pinto-Neto, Phys. Rev. D 78, 063506 (2008). arXiv:0809.2022

9. R.A. Leacock, M.J. Padgett, Phys. Rev. Lett. 50, 3 (1983)

10. R.A. Leacock, M.J. Padgett, Phys. Rev. D 28, 2491 (1983)

11. R.A. Leacock, M.J. Padgett, Am. J. Phys. 55, 261 (1986)

12. M.V. John, Found. Phys. Lett. 15, 329 (2002). arXiv:quant-ph/0109093

13. Gravitation and Cosmology, 21, 208 (2015). arXiv:1405.7957 [grqc]

14. C.D. Yang, Phys. Lett. A 372, 6253 (2008)

15. Chaos, Solitons Fractals 30, 342 (2006)

16. M. Rashki, S. Jalalzadeh, Phys. Rev. D 91, 023501 (2015). arXiv: 1412.3950
17. P. Frampton, S.D.H. Hsu, T.W. Kephart, D. Reeb, Class. Quantum Grav. 26, 145005 (2009). arXiv:0801.1847

18. J.J. Halliwell, Phys. Rev. D 38, 2468 (1988)

19. D. Wiltshire. arXiv:gr-qc/0101003

20. J.J. Halliwell. arXiv:0909.2566

21. N. Pinto-Neto, Found. Phys. 35, 577 (2005). arXiv:gr-qc/0410117

22. Y.V. Shtanov, Phys. Rev. D 54, 2564 (1996). arXiv:gr-qc/9503005

23. Q. Smith, The Monist 80, 160 (1997)

24. D.F. Styer et al., Am. J. Phys. 70, 288 (2002)

25. C. Tourenne, Ann. New York Acad. Sci. 480, 618 (1986)

26. C.-C. Chou, R.E. Wyatt, Phys. Rev. A 76, 012115 (2007)

27. C.-D. Yang, Ann. Phys. 321, 2876 (2006)

28. Y. Goldfarb, I. Degani, D.J. Tannor, J. Chem. Phys. 125, 231103 (2006)

29. R.E. Wyatt, Quantum Dynamics with Trajectories (Springer, Berlin, 2006)

30. A.S. Sanz and S. Miret-Artés, A Trajectory Description of Quantum Processes. I. Fundamentals, Springer Series: Lecture Notes on Physics (Springer, Berlin, 2012)

31. E.R. Floyd, Phys. Rev. D 26, 1339 (1982)

32. E.R. Floyd, Phys. Rev. D 25, 1547 (1982)

33. R. Colistete Jr., J.C. Fabris, N. Pinto-Neto, Phys. Rev. D 62, 083507 (2000). arXiv:gr-qc/0005013

34. F. Shojai, S. Molladavoudi, Gen. Rel. Grav. 39, 795 (2007). arXiv:0708.0620

35. P.S. Letelier, J.P.M. Pitelli, Phys. Rev. D 82, 104046 (2010). arXiv: 1010.3054

36. P. Pedram, S. Jalalzadeh, Phys. Rev. D 77, 123529 (2008). arXiv:0805.4099

37. P. Pedram, S. Jalalzadeh, S.S. Gousheh, Class. Quantum Grav. 24, 5515 (2007). arXiv:0709.1620

38. J. Marto, P.V. Moniz, Phys. Rev. D 65, 023516 (2001)

39. T. Rostami, S. Jalalzadeh, P.V. Moniz, Phys. Rev. D. 92, 023526 (2015). arXiv: 1507.04212

40. T. Rostami, S. Jalalzadeh, P.V. Moniz, Eur. Phys. J. C 75, 38 (2015). arXiv: 1412.6439

41. S. Jalalzadeh, P.V. Moniz, Phys. Rev. D 89, 083504 (2014). arXiv: 1403.2424

42. S. Jalalzadeh, T. Rostami, P.V. Moniz, Int. J. Mod. Phys. D 25, 1630009 (2016)

43. C. Kiefer, E. Joos, Lect. Notes Phys. 517, 105 (1999). arXiv:quant-ph/9803052

44. C.-D. Yang, Ann. Phys. 319, 399 (2005)

45. V. Mukhanov, Physical Foundations of Cosmology (Cambridge University Press, Cambridge, 2005)

46. S. Fay, T. Lehner, Gen. Rel. Grav. 37, 1097 (2005)

47. A. Perez, H. Sahlmann, D. Sudarsky, Class. Quant. Grav. 23, 2317 (2006). arXiv:gr-qc/0508100

48. N. Pinto-Neto, J.C. Fabris, Class. Quant. Grav. 30, 143001 (2013). arXiv: 1306.0820

49. N. Pinto-Neto, G. Santos, W. Struyve, Phys. Rev. D 89, 023517 (2014). arXiv:1309.2670

50. N. Pinto-Neto, G. Santos, W. Struyve, Phys. Rev. D 85, 083506 (2012). arXiv:1110.1339

51. R. Tumulka, Gen. Rel. Grav. 48, 2 (2016). arXiv: 1507.08542

52. S. Goldstein, W. Struyvey and R. Tumulka. arXiv:1508.0101 